\title{
Permanent Interstitial Brachytherapy for Previously Irradiated Head and Neck Cancer
}

William Breen ${ }^{1}$, Jacqueline Kelly ${ }^{1}$, Henry S. Park ${ }^{1}$, Yung Son ${ }^{1}$, Clarence Sasaki ${ }^{2}$, Lynn Wilson ${ }^{1}$, Roy Decker $^{1}$, Zain A. Husain ${ }^{1}$

1. Department of Therapeutic Radiology, Yale University School of Medicine, New Haven, USA 2. Otolaryngology, Yale University School of Medicine, New Haven, USA

Corresponding author: William Breen, wgbreen@gmail.com

\begin{abstract}
Objective: To evaluate our institutional experience using brachytherapy for the re-irradiation of the head and neck.
\end{abstract}

Study Design/Methods: We reviewed the records of patients who received brachytherapy for head and neck cancer in a previously irradiated field between 2007 and 2016.

Results: Sixty-nine patients received brachytherapy-based re-irradiation. Forty-nine patients (71\%) were treated for recurrent cancers, 15 patients (22\%) had second primary cancers, and five patients $(7 \%)$ were treated for persistent tumors. The median dose was $90 \mathrm{~Gy}$ (range 30-180). Median follow-up was 3.0 years for surviving patients and 0.6 years for all patients. Overall survival at one, three, and five years was $58 \%$, $19 \%$, and $12 \%$, respectively. Local control at one, three, and five years was $55 \%, 38 \%$, and $28 \%$, respectively. A disease-free interval of less than one year was associated with significantly worse local control $(\mathrm{p}=.04)$. Patients who received brachytherapy for a neck disease had significantly worse locoregional control than those who received brachytherapy for mucosal disease (heart rate (HR) 2.14, 95\% CI 1.00-4.56, $\mathrm{p}=.05$ ). Patients who had an extranodal extension had significantly worse overall survival than those without an extranodal extension (HR 2.57, 95\% CI 1.28-5.37, $\mathrm{p}=.008$ ). Seventy-four percent of patients who had pain before brachytherapy (with or without surgery) had an improvement of symptoms. Acute and chronic toxicity of at least Common Terminology Criteria for Adverse Events Grade 3 was seen in 27\% and 19\% of the patients, respectively.

Conclusions: Brachytherapy-based re-irradiation is an effective approach for patients undergoing reirradiation for head and neck cancer. Brachytherapy may be more effective for mucosal recurrences than neck recurrences.

Received 04/05/2018

Review began 04/10/2018

Review ended 04/20/2018

Published 04/22/2018

๑) Copyright 2018

Breen et al. This is an open access article distributed under the terms of the Creative Commons Attribution License CC-BY 3.0., which permits unrestricted use, distribution, and reproduction in any medium, provided the original author and source are credited.
Categories: Radiation Oncology, Oncology

Keywords: head and neck cancer, brachytherapy, re-irradiation, radiation, interstitial brachytherapy, oncology, palliative radiotherapy

\section{Introduction}

Patients with head and neck cancer frequently have disease recurrence, with significant locoregional failure rates for patients with advanced disease treated with chemoradiation [1-2]. Second primary cancers are also common and represent the second-most common cause of death in patients with head and neck cancer [3]. In either case, the management of head and neck cancer in a previously treated area is a therapeutic challenge. Former therapies limit further management options and increase the morbidity of any potential treatment. In the case of recurrent disease, the problem is compounded by the fact that the disease in question is likely treatment refractory. Still, there is value in selecting patients for aggressive curative intent interventions, as several series have demonstrated that a minority of patients will become long-term survivors [4-7].

While salvage surgery remains the standard of care for patients with recurrent disease in previously irradiated areas, recurrence rates are substantial following surgical resection alone, with reported rates ranging from $49 \%$ to over $80 \%$ [8-9]. As demonstrated in the landmark phase III study by Janot et al., diseasefree survival in these patients can be improved with chemoradiation [9]. The disease control benefits did come at a cost, however, with a two-year rate of grade three or four late toxicity of $39 \%$ in the chemoradiation arm compared to $10 \%$ in the "wait and see" arm.

Patients in that study were treated with 3-D conformal external beam radiation therapy. Given the sharper dose fall-off associated with brachytherapy, this therapy could potentially maintain similarly improved control rates while reducing long-term toxicity [10]. Unfortunately, the usage of brachytherapy has been declining in the definitive management of head and neck cancers [11]. Similarly, despite multiple studies of brachytherapy in the re-irradiation setting, it is not commonly used. 
Brachytherapy has long been used at our institution as part of the treatment approach for recurrent and new primary head and neck cancers arising in previously irradiated areas, and the purpose of this study is to explore the outcomes associated with brachytherapy-based re-irradiation in a modern series of patients.

\section{Materials And Methods}

This study was approved by our Institutional Review Board and was in accordance with the Helsinki Declaration of 1975 , revised in 2000 . The medical records of all patients treated with brachytherapy for head and neck cancers to a previously radiated area during the era of rigorous electronic medical records were retrospectively analyzed. Sixty-nine patients met each of the following criteria and were included:

1) Pathologically confirmed cancer of the head and neck treated with brachytherapy at our institution between 2007 and 2016.

2) Brachytherapy was applied to an area previously radiated with external beam radiation therapy (EBRT) to a dose greater than $45 \mathrm{~Gy}$.

3) All sites of known disease were addressed with treatment at the time of brachytherapy.

Patients were selected for brachytherapy at the discretion of individual physicians.

\section{Brachytherapy}

Brachytherapy was applied using the clinical judgment of the radiation oncologist. While preoperative imaging was used for guidance, no pre-operative dosimetry was performed. The radiation oncologist, with the help of the collaborating surgeon, sutured the brachytherapy meshes to the at-risk tissues, using either iodine-125 seeds or palladium-103 seeds. Post-operative CT scans and dosimetry were performed in the majority of cases $(n=38)$.

\section{Data collection}

Data collected included demographics, initial imaging, initial treatment, initial pathology, human papillomavirus (HPV) status, recurrence treatment, recurrence pathology, local, regional, and distant control, and survival.

Extranodal extension (ENE) was defined as cancer extending beyond the capsule of a lymph node and was assessed with initial pathology and at recurrence. Patients in whom neck disease obliterated any remaining lymph node structure were classified as having ENE. Overall survival (OS), local control (LC), and locoregional control (LRC) were calculated from the time of brachytherapy re-irradiation. The disease-free interval before brachytherapy was calculated from the most recent local treatment to brachytherapy reirradiation. Local control was defined as a lack of local failure. Local failure was defined as radiographic or clinical failure within two $\mathrm{cm}$ of the brachytherapy site. This method was chosen in order to differentiate patients who failed within the tissue directly treated by brachytherapy from those who failed outside of the brachytherapy treatment volume but within the region. Any failure elsewhere in the head and neck was considered a regional failure. Failures outside the head and neck were considered distant metastases. All sequential recurrence events were recorded (i.e., local, regional, and distant), not just initial events.

\section{Statistical analysis}

Statistical analyses were performed with the JMP software (SAS Institute Inc., Cary, North Carolina). Survival curves were created using the Kaplan-Meier method. Univariate Cox proportional hazards regression modeling was used to evaluate the association of patient and treatment factors with LC, LRC, and OS. Significance was defined as a p-value of 0.05 or less.

\section{Results}

Sixty-nine patients were identified. Patient characteristics appear in Table 1 . The median age at initial diagnosis was 58 (range 30-91). Fifty-seven (83\%) patients were male. Seventy-four percent of patients were smokers. Median pack-years was 30 (range 0-90). Sixty-five patients had squamous cell carcinoma, three had acinic cell carcinoma, and one had pleomorphic sarcoma. The initial T stage was most frequently T2 (40\%) and T3 (24\%). The initial N stage was most frequently N0 (44\%) and N2 (33\%). HPV status was known in only 14 patients (seven positive). For oropharyngeal cancers, HPV status was known in only eight of 21 patients (four positive). Forty-nine patients (71\%) were treated for new primaries, 15 (22\%) were treated for recurrences, and five (7\%) were treated for a persistent disease. 


\section{Cureus}

\section{Patients}

Median Age

Male

Female

Smoking History:

Oral Cavity Primary

Oropharynx Primary

Nasopharynx Primary

Larynx/Hypopharynx

Skin/Parotid

Unknown Primary

Brachytherapy for Recurrent Tumor

Brachytherapy for Second Primary

Brachytherapy for Persistent Tumor

Initial Surgery

Initial EBRT

Median Initial EBRT dose (range)

Initial Chemotherapy

Initial Brachytherapy

Brachytherapy for Mucosal Tumor

Brachytherapy for neck tumor

Brachytherapy for Mucosal and Neck Tumor

Salvage Surgery with Intraoperative Brachytherapy

Brachytherapy Alone

Systemic Therapy for Recurrence $n=69$

$58(30-91)$

$\mathrm{n}=57(83 \%)$

$\mathrm{n}=12(17 \%)$

$\mathrm{n}=46(74 \%)$

$n=24(35 \%)$

$\mathrm{n}=21(30 \%)$

$n=2(3 \%)$

$n=13(19 \%)$

$n=7(10 \%)$

$n=2(3 \%)$

49 (71\%)

15 (22\%)

$5(7 \%)$

$\mathrm{n}=46(67 \%)$

$\mathrm{n}=62(90 \%)$

63.6 Gy (45-76)

$\mathrm{n}=35(51 \%)$

$n=5(7 \%)$

$n=41(59 \%)$

$n=24(35 \%)$

$n=4(6 \%)$

$\mathrm{n}=58(84 \%)$

$\mathrm{n}=11(16 \%)$

$n=10(14 \%)$

TABLE 1: Patient and Treatment Characteristics

EBRT: external beam radiation therapy

\section{Previous treatments}

Please see Table 1 for treatment characteristics. All patients had a history of having received EBRT as part of a previous treatment course. Sixty-two patients had radiation at initial treatment; the remaining seven had radiation after recurrence. The median time between the first course of irradiation and brachytherapy was 1.8 years (range $0.12-24.8$ ).

\section{Brachytherapy for recurrence}

Permanent interstitial brachytherapy was used for mucosal disease in 41 patients (59\%), neck disease in 24 (35\%), and both mucosal and neck disease in four (6\%). Resection was performed at the time of brachytherapy (salvage surgery) in 58 patients (84\%), while brachytherapy alone was performed in 11 patients (16\%). Of the 28 patients who received neck brachytherapy, 27 (96\%) had neck dissection. Of the 41 patients who received brachytherapy to mucosal disease only, 17 (41\%) had neck dissection. The median brachytherapy activity was 32.4 millicuries (range 8.7-56.5). The median number of brachytherapy seeds used was 45 (range 6-95). Iodine-125 seeds were used in 43 patients (62\%), while palladium-103 seeds were used in 26 patients (38\%). Post-operative CT scans and dosimetry were performed in the majority of cases $(n=38)$. The median calculated brachytherapy dose to the tumor surface was $90 \mathrm{~Gy}$ (range 30-180) for these 


\section{Cureus}

patients.

\section{Outcomes}

OS at one, three, and five years was 58\%, 19\%, and 12\%, respectively (Figure 1). LC at one, three, and five years was $55 \%, 38 \%$, and $28 \%$, respectively (Figure 2). LRC at one, three, and five years was $50 \%$, $34 \%$, and $25 \%$, respectively. Median time to locoregional failure for patients who received brachytherapy for neck disease and those who received brachytherapy for mucosal sites alone was 0.6 and 1.2 years, respectively $(\mathrm{p}=.04)$ (Figure 3). There was a trend towards inferior OS in patients treated for neck disease (median OS 0.8 vs. 1.3 years, respectively), but this did not reach statistical significance $(p=.06)$ (Figure 4$)$. Median OS for patients with ENE at the time of brachytherapy was 0.6 years, compared to 1.3 years for patients without ENE at the time of brachytherapy ( $\mathrm{p}=.007$ ) (Figure 5). Twenty-four of 44 (54\%) patients who had lymph nodes dissected at recurrence had ENE. Fifteen of 59 (25\%) patients had less than an R0 resection. Patients with an R1 resection had significantly worse OS than those with an R0 resection (RR 2.46, 95\% CI 1.22-4.81, $\mathrm{p}=.01)$. No patients had an $\mathrm{R} 2$ resection.

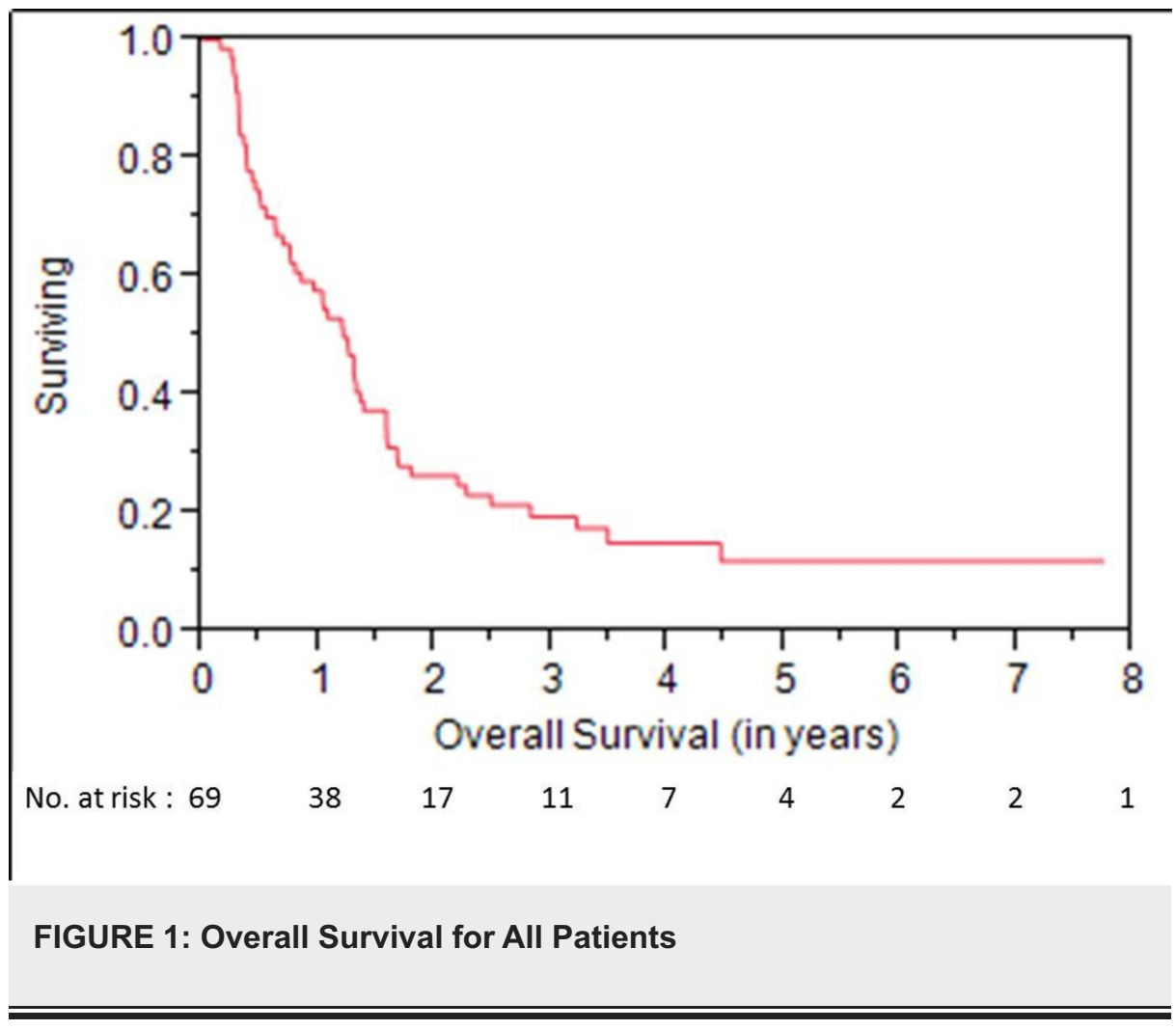




\section{Cureus}

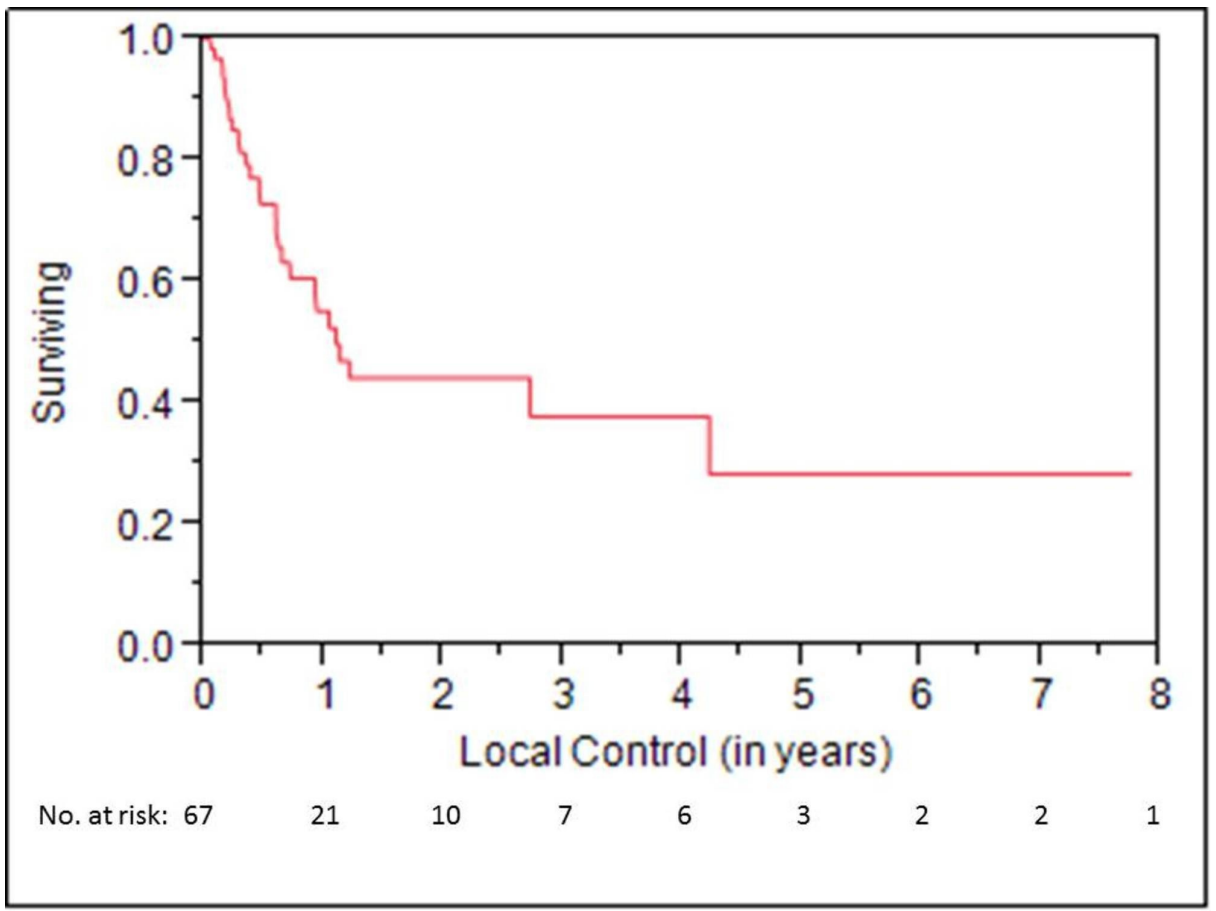

FIGURE 2: Local Control for All Patients

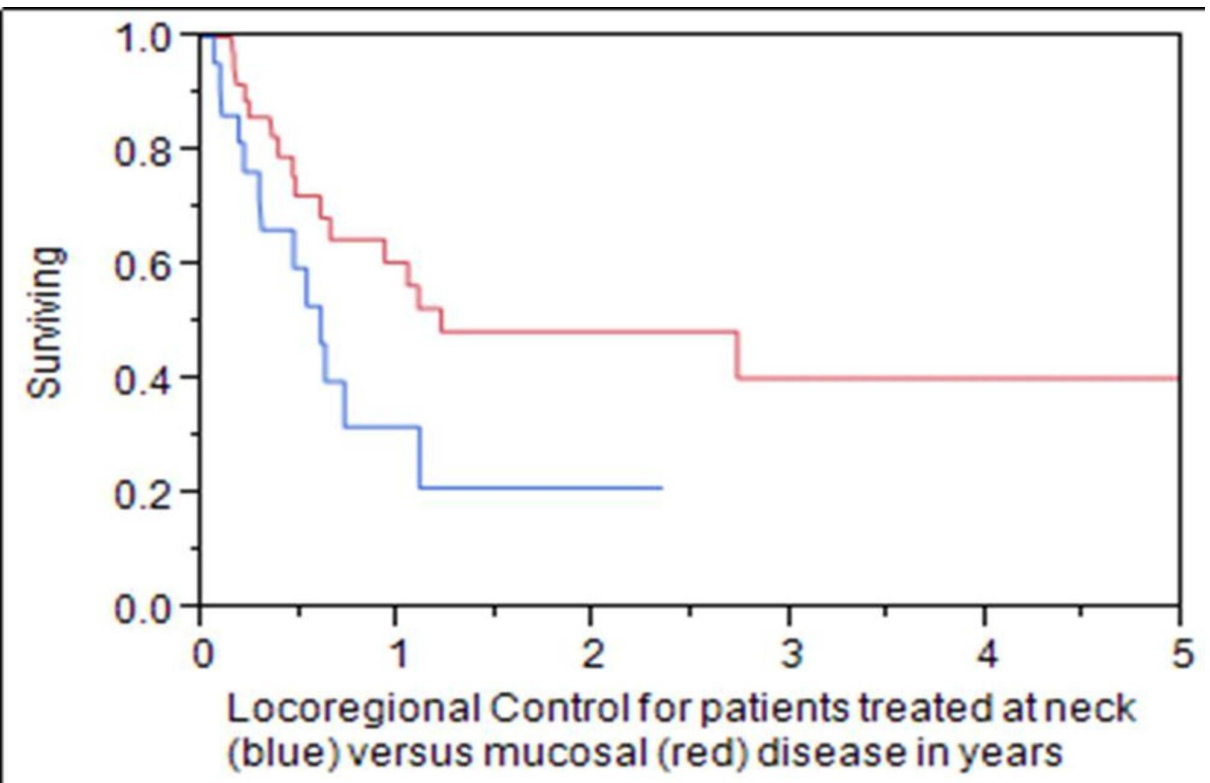

No. at risk

Mucosal: 39

Neck:

16

8

6

5

3

FIGURE 3: Locoregional Control for Patients Treated at Neck versus Mucosal Disease in Years

Blue line $=$ neck disease 


\section{Cureus}

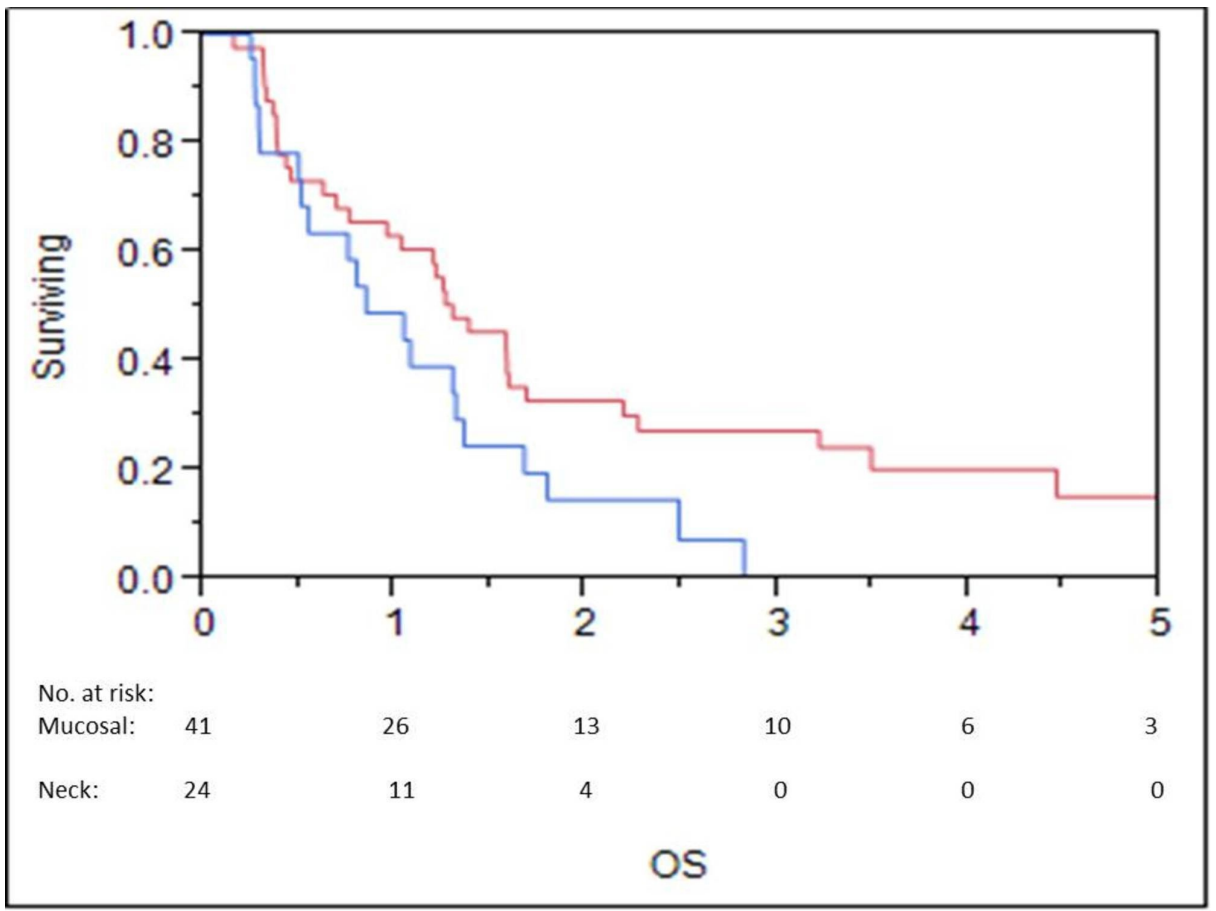

FIGURE 4: Overall Survival for Mucosal versus Neck Disease

Red line $=$ mucosal disease

Blue line $=$ neck disease

OS: overall survival

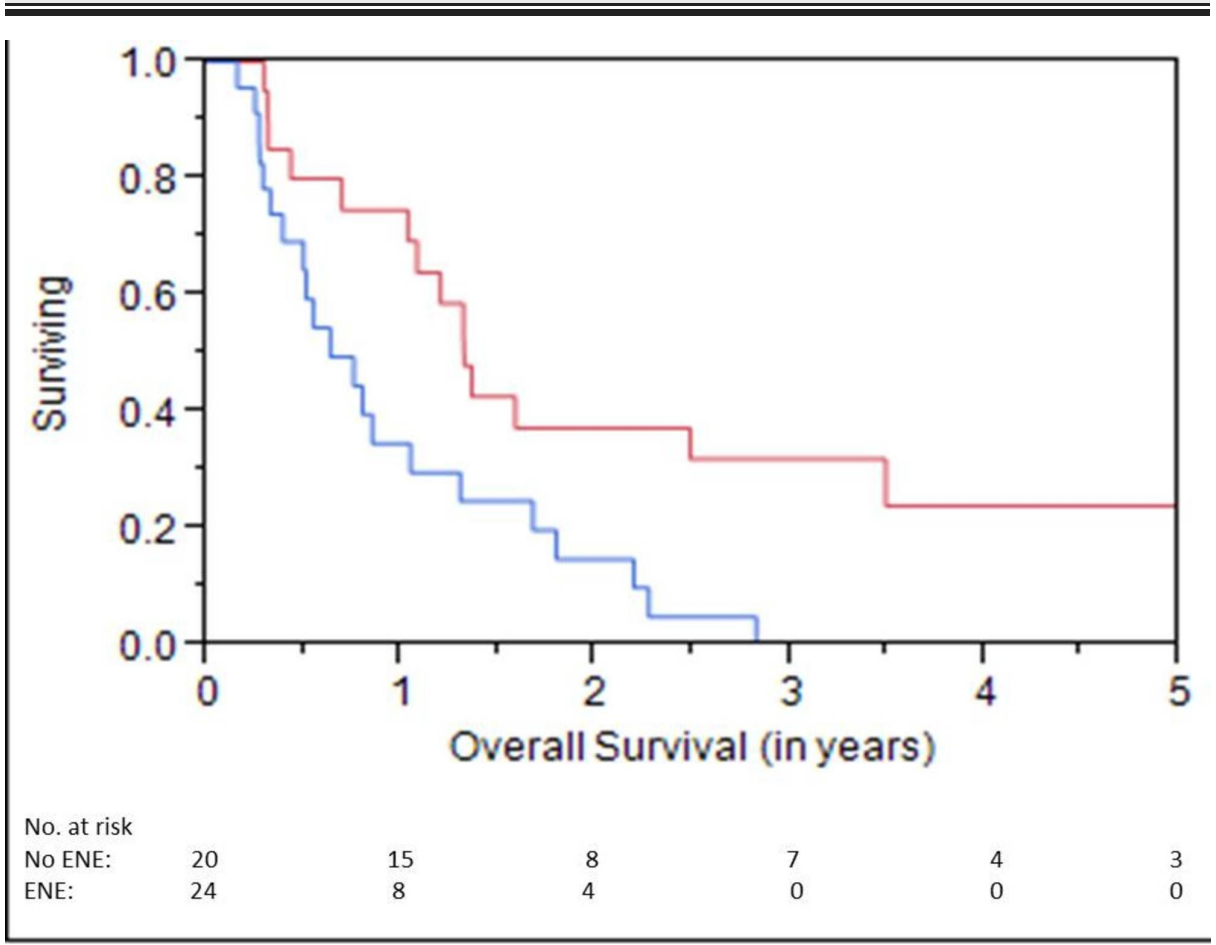

FIGURE 5: Overall Survival for ENE

Blue Line $=$ ENE present

Red Line $=$ ENE absent

ENE: extranodal extension 


\section{Cureus}

Seven patients (10\%) developed distant metastasis at a median time of 0.46 years after brachytherapy (Table 2).

\section{Median Overall Survival (years)}

1 year OS

3 year OS

5 year OS

Median time to local failure

1 year LC

3 year LC

5 year LC

Median time to regional failure

1 year LRC

3 year LRC

5 year LRC

Developed distant metastasis?

Palliation

Had pain before brachytherapy

Pain improved after treatment

Acute CTCAE toxicities:

Any grade 4:

Any grade 3 :

Grade 3 dyspagia or xerostomia

Any grade 2 or 3 :

Chronic CTCAE toxicities:

Any grade 4:

Grade 4 trismus

Grade 4 fibrosis

Any grade 3:

Grade 3 dysphagia

Grade 3 fibrosis

Grade 3 osteonecrosis

Any grade 3-4:
$1.2(.03-7.7)$

$58 \%$

$19 \%$

$12 \%$

$1.1(.05-4.1)$

$55 \%$

$38 \%$

$28 \%$

$0.9(.05-4.1)$

$50 \%$

$34 \%$

$25 \%$

$\mathrm{n}=7(10 \%)$

$\mathrm{n}=35$

$\mathrm{n}=26(74 \%)$ $\mathrm{n}=0$

$\mathrm{n}=18(27 \%)$

$\mathrm{n}=18(27 \%)$

$n=48(72 \%)$

$\mathrm{n}=2(3 \%)$

$\mathrm{n}=1(2 \%)$

$\mathrm{n}=1(2 \%)$

$n=12(19 \%)$

$\mathrm{n}=7(11 \%)$

$\mathrm{n}=2(3 \%)$

$n=2(3 \%)$

$\mathrm{n}=14(22 \%)$ 


\section{Cureus}

\section{TABLE 2: Oncologic Outcomes and Toxicities}

OS: overall survival

LC: local control

LRC: locoregional control

CTCAE: common terminology criteria for adverse events

Forty-seven (68\%) of patients received brachytherapy for the first recurrence, while the remaining 22 (32\%) received brachytherapy for the second or greater recurrence (Table 1). There was no difference in OS between patients being treated for the first recurrence versus patients treated for the second or greater recurrence (RR 1.04, 95\% CI .60-1.88, p=.89). A time to first recurrence of less than one year was not associated with significantly worse OS (RR 0.82, 95\% CI .44-1.47, p=.52) or local control (RR 1.01, 95\% CI .42$2.25, \mathrm{p}=0.98)$. A disease-free interval of greater than one year before brachytherapy was associated with significantly improved local control (63\% vs. 39\% at one year, $\mathrm{p}=.0437)$, but not OS (69\% vs. $31 \%$ at one year, $\mathrm{p}=0.25$ )

\section{Palliation}

Of the 35 patients with uncontrolled pain before treatment, 26 (74\%) had improvement in their pain following treatment. Of the 26 who had improvement in pain, 21 received intraoperative brachytherapy (IOBT) at the time of resection, while the remaining five had successful palliation after receiving brachytherapy alone.

\section{Adverse events}

Adverse events are listed in Table 2. A carotid rupture occurred in five patients. Four of these patients had mucosal sites treated with brachytherapy, and two were treated at neck sites (one patient treated at both mucosal and neck sites). All five patients who had carotid rupture had neck dissection at the time of brachytherapy. For three of these patients, stripping of the carotid artery was specifically mentioned in the operative note. Furthermore, in these cases, the brachytherapy mesh was applied directly over the carotid artery as part of the resection bed. Median time from brachytherapy to rupture was 118 days. Two patients had the rupture in the first two weeks after surgery. After the carotid rupture, median survival was 92 days. One patient died the day of the rupture, and another died three days later. One patient with rupture is still alive at long-term follow-up.

\section{Discussion}

In this modern cohort of patients, we were able to demonstrate favorable outcomes associated with brachytherapy-based re-irradiation of head and neck cancers. Several notable findings emerged from this study, which may help determine which patients benefit most from this approach.

The one-year OS of $58 \%$ is consistent with that seen in the literature, although variability in patient selection makes comparisons difficult [12-13]. More importantly, the three-year OS of 19\% again suggests that a minority of patients will be long-term survivors, indicating a utility to this approach. However, this survival is inferior compared to the $20 \%$ five-year survival reported in patients treated with EBRT reirradiation in a recent study from Bots et al. [14]. Patient selection may have contributed to this difference, as the patients included in our study often were not felt to be candidates for further EBRT. The one-year LRC rate of $50 \%$ that we report is also generally consistent with what is seen in the literature [12-13].

Importantly, there was a substantial difference in LRC in patients treated for primary mucosal tumors versus those treated for neck disease ( 1.2 vs 0.6 years, respectively, $\mathrm{p}=.04$ ), as well as a trend towards worse survival in patients with neck recurrences (OS 1.3 vs. 0.8 years, $p=.06$ ). These findings must be considered as only hypothesis generating, as a multivariate analysis was not performed. This area needs further study to examine whether the risk of microscopic infiltration in the neck is more significant than in the primary tumor sites, which could mean that irradiating a larger area of the neck with brachytherapy may prove more beneficial. Additionally, patients with ENE in the neck did particularly poorly, with no long-term survivors; brachytherapy in these patients was essentially local palliation.

Our data also support the importance of LC in this poor prognosis group of patients. Only $10 \%$ of patients developed distant metastases, reaffirming the importance of local disease control in the head and neck. This is less than the $27 \%$ rate of distant failure in this set of patients reported by Salama et al. [15]. Additionally, 
our data also suggest a palliative benefit in terms of pain. Of the patients who were noted to have pain before treatment, nearly $75 \%$ saw an improvement in pain with therapy, which was predominantly resection with brachytherapy.

Our results also confirm the findings of other studies, namely, that patients treated with less than a oneyear interval between courses of irradiation had a trend towards worse control and survival outcomes $[7,16]$. A report by Teckie et al., which also looked at patients receiving IOBT for recurrent head and neck cancers, similarly showed worse LC with a disease-free interval shorter than one year [17].

Our results did show the expected toxicity profile with $22 \%$ of the patients experiencing grade three or four late toxicity [13]. Notably, there was a substantial risk of carotid rupture (also called carotid blowout) with five ruptures (7\%) among the group, higher than the $2.6 \%$ rate previously reported by McDonald et al. [18]. This relatively high rate can likely be attributed to the associated surgical stripping of the carotid artery before the application of brachytherapy, sometimes directly to the adventitia of the vessel. In three of these cases, the disease had to be stripped from the carotid, which likely contributed to the risk as well, especially in light of the fact that two of the carotid ruptures occurred within two weeks of surgery, at which time, only a modest amount of radiation had been delivered. Still, these results highlight the risks of this approach. More recently, our group's approach has changed to place a flap of tissue above the carotid vessels, in between the brachytherapy seeds, in the hope that this may decrease the risk of injury.

The largest series utilizing low-dose-rate (LDR) brachytherapy to date was performed by Puthawala et al., who reviewed the records of 220 patients treated between 1979 and 1997 for the recurrence of previously irradiated head and neck cancers [13]. At a minimum six month follow-up, local tumor control was achieved in $77 \%(217 / 282)$ of the implanted tumor sites. The two- and five-year disease-specific survival rates for the entire group were $60 \%$ and $33 \%$, respectively. The OS for the entire group at five years was $21.7 \%$. Moderate to severe late complications occurred in $27 \%$ of the patients. Our study had no minimum follow-up, in order to assess for peri-operative mortality and early failure, and did not have a maximum disease size, which likely led to the difference in OS.

More recently, Grimard et al. published results of 45 patients treated with LDR brachytherapy as the primary or adjuvant treatment for the first recurrence of head and neck cancer [12]. LC at one and two years was 50\% and $37 \%$, respectively. Median survival after brachytherapy was 16 months.

The strengths of the current study include its modern patient cohort, the inclusion of patients without a minimum follow-up, and the assessment of palliative outcomes. There are a number of weaknesses of this small, retrospective study that must be acknowledged, including patient selection bias, limitations assessing toxicities, uneven follow-up, and changing treatment paradigms. This data is from one academic center, and the results may not apply to other settings. HPV status was unknown for the majority of patients. The tumors and treatments used were heterogeneous. Three acinic cell carcinoma and one pleomorphic sarcoma were included. Brachytherapy was applied using the judgment of the radiation oncologist and without preoperative dosimetry, resulting in varying doses and incomplete treatment information. Dosing information was only available for $55 \%$ of patients, making an assessment of the optimal brachytherapy dose unfeasible. Finally, the statistical methods used were less than optimal. Competing risks methodology could not be used because of the limitations of the dataset. Only a univariate analysis was performed, so all results must be considered hypothesis generating rather than definitive.

\section{Conclusions}

Our results suggest that brachytherapy is a viable option for certain cases of head and neck cancers in previously irradiated areas. Specifically, our data indicate that brachytherapy may be better suited for mucosal disease than neck disease, particularly in patients with known ENE who have especially poor outcomes. Future directions for research include prospective data collection on dosing, toxicities, and outcomes associated with brachytherapy for head and neck cancers in previously radiated areas.

\section{Additional Information}

\section{Disclosures}

Human subjects: Consent was obtained by all participants in this study. Yale School of Medicine/YNNH IRB issued approval 100FR7(2016-2). This study was approved by our Institutional Review Board and was in accordance with the Helsinki Declaration of 1975, revised in 2000. Animal subjects: All authors have confirmed that this study did not involve animal subjects or tissue. Conflicts of interest: In compliance with the ICMJE uniform disclosure form, all authors declare the following: Payment/services info: All authors have declared that no financial support was received from any organization for the submitted work. Financial relationships: All authors have declared that they have no financial relationships at present or within the previous three years with any organizations that might have an interest in the submitted work. Other relationships: All authors have declared that there are no other relationships or activities that could appear to have influenced the submitted work. 


\section{References}

1. Brockstein B, Haraf DJ, Rademaker AW, et al.: Patterns of failure, prognostic factors and survival in locoregionally advanced head and neck cancer treated with concomitant chemoradiotherapy: a 9-year, 337patient, multi-institutional experience. Ann Oncol. 2004, 15:1179-1186. 10.1093/annonc/mdh308

2. Blanchard P, Baujat B, Holostenco V, Bourredjem A, Baey C, Bourhis J, Pignon JP: Meta-analysis of chemotherapy in head and neck cancer (MACH-NC): a comprehensive analysis by tumour site. Radiother Oncol. 2011, 100:33-40. 10.1016/j.radonc.2011.05.036

3. Baxi SS, Pinheiro LC, Patil SM, Pfister DG, Oeffinger KC, Elkin EB: Causes of death in long-term survivors of head and neck cancer. Cancer. 2014, 120:1507-1513. 10.1002/cncr.28588

4. Lee N, Chan K, Bekelman JE, et al.: Salvage re-irradiation for recurrent head and neck cancer . Int J Radiat Oncol Biol Phys. 2007, 68:731-740. 10.1016/j.ijrobp.2006.12.055

5. Popovtzer A, Gluck I, Chepeha DB, et al.: The pattern of failure after reirradiation of recurrent squamous cell head and neck cancer: implications for defining the targets. Int J Radiat Oncol Biol Phys. 2009, 74:13421347. 10.1016/j.ijrobp.2008.10.042

6. Riaz N, Hong JC, Sherman EJ, et al.: A nomogram to predict loco-regional control after re-irradiation for head and neck cancer. Radiother Oncol. 2014, 111:382-387. 10.1016/j.radonc.2014.06.003

7. Spencer SA, Harris J, Wheeler RH, et al.: Final report of RTOG 9610, a multi-institutional trial of reirradiation and chemotherapy for unresectable recurrent squamous cell carcinoma of the head and neck. Head Neck. 2008, 30:281-288. 10.1002/hed.20697

8. Goodwin Jr. WJ: Salvage surgery for patients with recurrent squamous cell carcinoma of the upper aerodigestive tract: when do the ends justify the means?. Laryngoscope. 2000, 110:1-18. 10.1097/00005537200003001-00001

9. Janot F, de Raucourt D, Benhamou E, et al.: Randomized trial of postoperative reirradiation combined with chemotherapy after salvage surgery compared with salvage surgery alone in head and neck carcinoma. J Clin Oncol. 2008, 26:5518-5523. 10.1200//CO.2007.15.0102

10. Mazeron JJ, Ardiet JM, Haie-Meder C, et al.: GEC-ESTRO recommendations for brachytherapy for head and neck squamous cell carcinomas. Radiother Oncol. 2009, 91:150-156. 10.1016/j.radonc.2009.01.005

11. Orton A, Boothe D, Gan M, Monroe MM, Hitchcock YJ, Lloyd S: The "decay" of brachytherapy use in tumors of the oral cavity: a population-based patterns of care and outcomes analysis from 1973 to 2012. Brachytherapy. 2016, 15:851-858. 10.1016/j.brachy.2016.05.007

12. Grimard L, Esche B, Lamothe A, Cygler J, Spaans J: Interstitial low-dose-rate brachytherapy in the treatment of recurrent head and neck malignancies. Head Neck. 2006, 28:888-895. 10.1002/hed.20422

13. Puthawala A, Nisar Syed AM, Gamie S, Chen YJ, Londrc A, Nixon V: Interstitial low-dose-rate brachytherapy as a salvage treatment for recurrent head-and-neck cancers: long-term results. Int J Radiat Oncol Biol Phys. 2001, 51:354-362. 10.1016/S0360-3016(01)01637-6

14. Bots WTC, van den Bosch S, Zwijnenburg EM, et al.: Reirradiation of head and neck cancer: long-term disease control and toxicity. Head Neck. 2017, 6:1122-1130. 10.1002/hed.24733

15. Salama JK, Vokes EE, Chmura SJ, et al.: Long-term outcome of concurrent chemotherapy and reirradiation for recurrent and second primary head-and-neck squamous cell carcinoma. Int J Radiat Oncol Biol Phys. 2006, 64:382-391. 10.1016/j.ijrobp.2005.07.005

16. Lee JY, Suresh K, Nguyen R, et al.: Predictors of severe long-term toxicity after re-irradiation for head and neck cancer. Oral Oncol. 2016, 60:32-40. 10.1016/j.oraloncology.2016.06.017

17. Teckie S, Scala LM, Ho F, et al.: High-dose-rate intraoperative brachytherapy and radical surgical resection in the management of recurrent head-and-neck cancer. Brachytherapy. 2013, 12:228-234. 10.1016/j.brachy.2013.01.165

18. McDonald MW, Moore MG, Johnstone PA: Risk of carotid blowout after reirradiation of the head and neck: a systematic review. Int J Radiat Oncol Biol Phys. 2012, 82:1083-1089. 10.1016/j.ijrobp.2010.08.029 